Article

\title{
Tunable Broadband Nonlinear Optical Properties of Black Phosphorus Quantum Dots for Femtosecond Laser Pulses
}

\author{
Xiao-Fang Jiang ${ }^{1}$, Zhikai Zeng ${ }^{1}$, Shuang Li $^{1}$, Zhinan Guo ${ }^{2}$, Han Zhang ${ }^{2}$, Fei Huang ${ }^{1, *}$ and \\ Qing-Hua $\mathrm{Xu}$ 2,3,4,* \\ 1 State Key Laboratory of Luminescent Materials and Devices, Institute of Polymer Optoelectronic Materials \\ and Devices, South China University of Technology, Guangzhou 510640, China; \\ msxfjiang@scut.edu.cn (X.-F.J.); zeng.zhikai@mail.scut.edu.cn (Z.Z.); mssli@scut.edu.cn (S.L.) \\ 2 SZU-NUS Collaborative Innovation Center for Optoelectronic Science and Technology, and Key Laboratory \\ of Optoelectronic Devices and Systems of Ministry of Education and Guangdong Province, \\ Shenzhen University, Shenzhen 518060, China; guozhinan@126.com (Z.G.); hzhang@szu.edu.cn (H.Z.) \\ 3 National University of Singapore (Suzhou) Research Institute, Suzhou 215123, China \\ 4 Department of Chemistry, National University of Singapore, Singapore 117543, Singapore \\ * Correspondence: msfhuang@scut.edu.cn (F.H.); chmxqh@nus.edu.sg (Q.-H.X.)
}

Academic Editor: Irene D'Amico

Received: 6 January 2017; Accepted: 16 February 2017; Published: 21 February 2017

\begin{abstract}
Broadband nonlinear optical properties from 500 to $1550 \mathrm{~nm}$ of ultrasmall black phosphorus quantum dots (BPQDs) have been extensively investigated by using the open-aperture Z-scan technique. Our results show that BPQDs exhibit significant nonlinear absorption in the visible range, but saturable absorption in the near-infrared range under femtosecond excitation. The calculated nonlinear absorption coefficients were found to be $(7.49 \pm 0.23) \times 10^{-3},(1.68 \pm 0.078) \times 10^{-3}$ and $(0.81 \pm 0.03) \times 10^{-3} \mathrm{~cm} / \mathrm{GW}$ for 500,700 and $900 \mathrm{~nm}$, respectively. Femtosecond pump-probe measurements performed on BPQDs revealed that two-photon absorption is responsible for the observed nonlinear absorption. The saturable absorption behaviors observed at 1050, 1350 and $1550 \mathrm{~nm}$ are due to ground-state bleaching induced by photo-excitation. Our results suggest that BPQDs have great potential in applications as broadband optical limiters in the visible range or saturable absorbers in the near-infrared range for ultrafast laser pulses. These ultrasmall BPQDs are potentially useful as broadband optical elements in ultrafast photonics devices.
\end{abstract}

Keywords: black phosphorus; quantum dots; nonlinear optics; two-photon absorption; femtosecond laser

\section{Introduction}

The upsurge of two-dimensional (2D) materials was triggered by the discovery of graphene in 2004 when it was isolated from its parent graphite [1]. Due to their outstanding physical and chemical properties and promising applications in various fields such as photovoltaics $[2,3]$ and electronics [4,5], 2D materials became a new class of nanomaterials that have a groundbreaking impact on nanotechnology. Current studies on 2D materials mainly focus on graphene and wide-bandgap transitional metal dichalcogenides (TMDs) such as molybdenum disulfide $\left(\mathrm{MoS}_{2}\right)$ [6] Most importantly, there is significant interest in studying 2D materials' optical properties and their applications as nonlinear optical materials (optical limiters and saturable absorbers). Graphene and graphene oxide have been reported to display broadband optical-limiting properties due to strong two-photon absorption [7-10]. Broadband nonlinear absorption was also discovered in TMDs [11,12] and their applications in ultrafast lasers have been investigated $[13,14]$. Besides 2D materials, ultrasmall 
quantum dots (QDs) are being also extensively studied due to their unique electronic and optical properties arising from the quantum confinement effect [15].

Black phosphorous (BP), also known as phosphorene, is the latest member of the family of $2 \mathrm{D}$ materials $[16,17] . \mathrm{BP}$ is the most thermodynamically stable allotrope of phosphorus [18] and its unique structure as well as fascinating optical and electronic properties have attracted a lot of research interests [19]. As bulk BP consists of phosphorene monolayers stacked together by van der Waals force, it can be mechanically [20] or chemically exfoliated [21-23] into few-layer or single-layer nanosheets, or quantum dots. Due to its attractive properties, BP has been applied to field-effect transistors (FET) [17,24]. Few-layer BP can be used in thin-film solar cells [25] and p-n diodes [26]. It is also theoretically predicted to be used in flexible ambipolar transistors [27], energy storage [28] and moveable vibratory devices [29]. In particular, it has been previously reported that few-layer $\mathrm{BP}$ can act as an effective saturable absorber for ultrashort pulse generation in solid-state and fiber mode-locked lasers operating in the 1000-2000 nm wavelength range [30-35].

Unlike graphene's zero-bandgap nature which limits its electronic and photonic applications, it is noteworthy that the bandgap of BP depends on the number of layers, ranging from 0.3 (bulk) to $2.0 \mathrm{eV}$ (single layer) [36,37]. It has also been found that the applied strain force [38,39], stacking order [25] and external electric field [40] can also modulate the bandgap of BP. The tunable bandgap of BP shows great potential in bridging the space between zero-bandgap semi-metallic graphene and wide-bandgap TMDs (1-2 eV). Hence, BP is considered to be suitable for extremely broadband nonlinear optical applications, including optical limiters and saturable absorbers [30-35]. The past decades have witnessed significant research efforts in developing broadband optical materials. Optical-limiting materials exhibit decreased transmittance at high-input laser intensity, which can be used to protect human eyes and sensitive instruments from damage by high-intensity laser beams [41]. In contrast, saturable absorbers show an increased transmittance at high-input laser intensity, which can be utilized in pulse compression, mode locking and Q-switching [42].

Herein, for the first time, black phosphorus quantum dots (BPQDs) are extensively investigated in broadband femtosecond nonlinear optical properties from visible to near-infrared (near-IR) range. Based on the simple solution exfoliation method, a suspension of BPQDs was prepared in N-Methylpyrrolidone solvent (NMP). The utrasmall BPQDs were experimentally demonstrated to display nonlinear optical responses in a broad wavelength range by femtosecond open-aperture Z-scan measurements. Under femtosecond laser excitation, BPQDs exhibited significant nonlinear absorption in the visible range, but saturable absorption in the near-infrared (near-IR) range. Femtosecond pump-probe measurements performed on BPQDs revealed that two-photon absorption is primarily responsible for the observed nonlinear absorption. The results suggest that BPQDs have great potential in applications as broadband optical limiters in the visible range or as saturable absorbers in the near-IR range for ultrafast laser pulses. These ultrasmall BPQDs are potentially useful as broadband optical elements in fiber lasers and other ultrafast photonics devices.

\section{Results and Discussion}

\subsection{Sample Preparation and Characterizations}

Mechanical and liquid exfoliation methods are commonly used as simple and effective techniques to prepare 2D nanomaterials and QDs from bulk crystals, such as BP [21,22] and graphene [43]. In the simple liquid exfoliation technique, solvents with a suitable surface energy can serve as stable dispersions for layered materials. Here in this article, the solvent exfoliation combined with probe sonication and bath sonication were used to fabricate BPQDs dispersed in N-Methylpyrrolidone (NMP) solvent [44]. BP has been known to be sensitive to water and oxygen and can be oxidized under visible-light irradiation [45,46]. In our experiments, BPQDs were prepared in ambient conditions and dispersed in NMP solution to avoid any exposure to air as much as possible. The absorption and Raman spectra were measured and compared before and after nonlinear optical property 
measurements to check for any possible degradation. More experimental details can be found in Section 3. Transmission electron microscopy (TEM) measurements were conducted to examine the morphology of the as-prepared BPQDs. The TEM image (Figure 1a) shows that the ultrasmall BPQDs have an average lateral size of about $2-3 \mathrm{~nm}$, which corresponds to the stacking number of layers of $2 \pm 1$. The absorption spectra (Figure $1 \mathrm{~b}$ ) show that BPQDs have a broad absorption band, spanning from the UV to near-IR range. The absorption band at the UV-visible range is similar to those of other 2D layered materials such as graphene oxide, as well as few-layer BP. Compared to BP, a huge absorption band in the near-IR range from 1250 to $1630 \mathrm{~nm}$ with two absorption maxima at 1438 and $1550 \mathrm{~nm}$ was observed in the BPQD dispersion. This near-IR broad absorption band might be due to the defect state absorption induced by the ultrasmall size of the QDs [47], which indicates possible interesting nonlinear optical properties of BPQDs both in visible and near-IR regions.

(a)

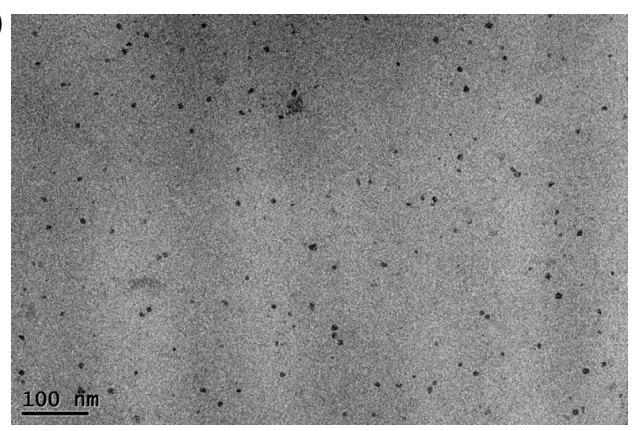

(b)

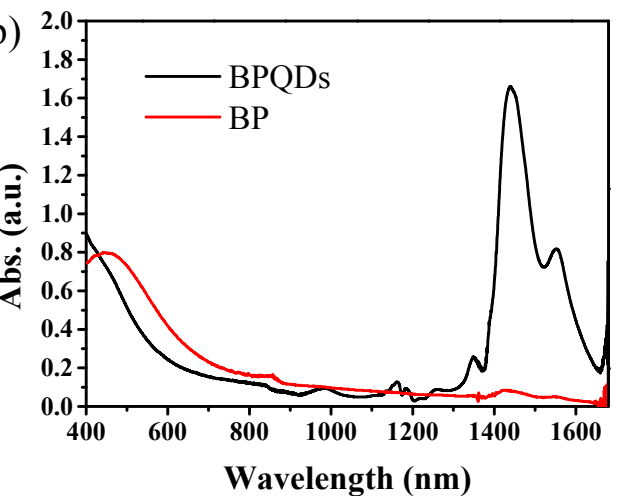

Figure 1. (a) TEM image of BPQDs; (b) absorption spectra of BP and BPQDs.

BPQDs were also characterized by Raman spectroscopy. Samples were prepared by spin-coating the BPQD dispersion onto the quartz substrates, drying them on a heating plate under $348 \mathrm{~K}$ for 5 min, and then keeping them in $\mathrm{N}_{2}$ for the next $6 \mathrm{~h}$. As shown in Figure 2, the three observed peaks can be attributed to one out-of-plane phonon mode $\left(\mathrm{A}_{\mathrm{g}}^{1}\right)$ at $361.16 \mathrm{~cm}^{-1}$, and two in-plane modes, which are $B_{2 g}$ and $A_{g}^{2}$, at 438.22 , and $465.65 \mathrm{~cm}^{-1}$, respectively, consistent with the reported values [15]. Compared to the bulk BP, both $B_{2 g}$ and $A_{g}^{2}$ modes of BPQDs are red-shifted by $2.30 \mathrm{~cm}^{-1}$, while the $A_{g}^{1}$ mode is red-shifted by $2.32 \mathrm{~cm}^{-1}$. It can be explained that the Raman shift is dependent on thickness and lateral dimensions. The frequency difference between the $\mathrm{A}_{\mathrm{g}}^{1}$ and $\mathrm{A}_{\mathrm{g}}^{2}$ modes of BPQDs equals $104.3 \mathrm{~cm}^{-1}$, which is larger than the reported value of bulk BP [48] and confirms that we successfully reduced the thickness of the BP.

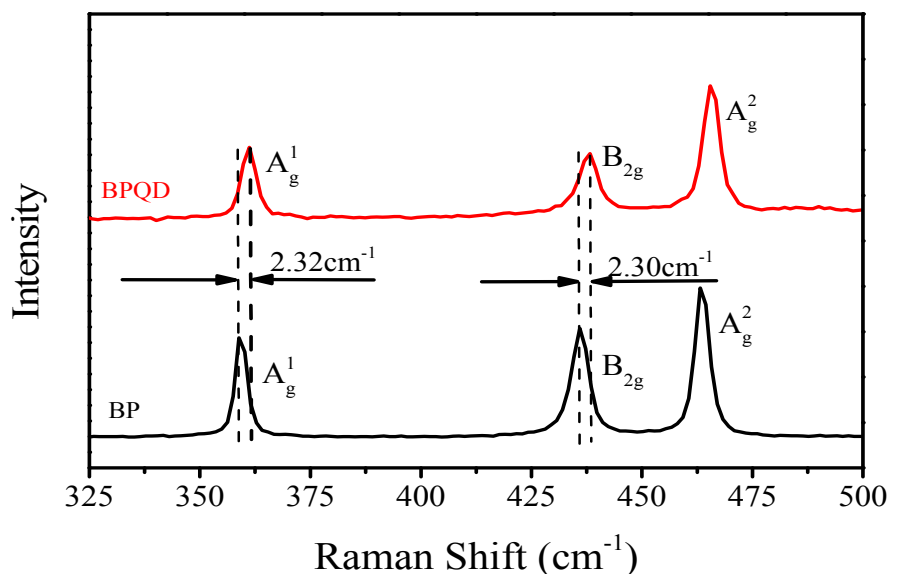

Figure 2. Raman spectra of BPQDs and bulk BP. 


\subsection{The Nonlinar Optical Properties of BPQDs in Visible Range}

Open-aperture femtosecond Z-scan measurements were used to characterize the nonlinear optical properties of BPQDs. The BPQD dispersion was placed in a $1 \mathrm{~mm}$ cuvette for Z-scan measurements. The detailed experimental setup is described in Section 3. Briefly, femtosecond laser pulses with a pulse duration of $120 \mathrm{fs}$ and a repetition rate of $1 \mathrm{kHz}$ were focused onto the cuvette with the samples, which were moved towards and away from the focus by using a motorized translational stage to study the excitation power intensity-dependent transmission of samples. Similar Z-scan measurements were performed with femtosecond laser pulses at 500, 700 and $900 \mathrm{~nm}$, which were generated by an optical parametric amplifier (TOPAS-Prime) pumped by a mode-locked Ti:sapphire oscillator-seeded regenerative amplifier. The Z-scan results showed that a very weak saturable absorption effect was exhibited in the visible spectra range upon low excitation intensity due to ground-state bleaching. As the excitation intensity increased, reverse saturable absorption occurred and finally became dominant. The results under the high excitation peak power intensity of $147 \mathrm{GW} / \mathrm{cm}^{2}$ are shown in Figure 3. The subfigures $(\mathrm{a}-\mathrm{c})$ are corresponding to the Z-scan results at $500 \mathrm{~nm}(\mathrm{a}), 700 \mathrm{~nm}(\mathrm{~b})$ and $900 \mathrm{~nm}$ (c), respectively. NMP solvent in a $1 \mathrm{~mm}$ cuvette was measured and no nonlinear absorption was found, which excludes the effects of NMP and the $1 \mathrm{~mm}$ cuvette. For all wavelengths, the normalized transmittance gradually decreased as the sample move towards the focus point $(Z=0)$, indicating an optically induced reverse saturable absorption. The Z-scan curves can be fitted to achieve the nonlinear absorption coefficient $\beta$ with the following approximate equation:

$$
T(Z)=\sum_{m=0}^{\infty}\left(-\beta I_{0} L_{e f f}\right)^{m} /\left(1+Z^{2} / Z_{0}^{2}\right)^{m}(m+1)^{3 / 2} \approx 1-\beta I_{0} L_{e f f} / 2^{3 / 2}\left(1+Z^{2} / Z_{0}^{2}\right)
$$

where $Z$ is the distance between the sample and the focus; $Z_{0}$ is the Rayleigh diffraction length; $I_{0}$ is the peak power density of the excitation laser pulses; $L_{e f f}=\left(1-\mathrm{e}^{-\alpha_{0} L}\right) / \alpha_{0}$ is the effective sample length; $L$ is the sample length; $\alpha_{0}$ is the linear absorption coefficient. The fitting values of nonlinear absorption coefficient $\beta$ at 500,700 and $900 \mathrm{~nm}$ were found to be $(7.49 \pm 0.23) \times 10^{-3},(1.68 \pm 0.078) \times 10^{-3}$ and $(0.81 \pm 0.03) \times 10^{-3} \mathrm{~cm} / \mathrm{GW}$ for BPQDs, respectively. The obtained nonlinear absorption coefficients of these BPQDs are higher than those of other optical-limiting materials such as CdSe QDs with average size of around $2 \mathrm{~nm}\left((1.1 \pm 0.15) \times 10^{-3} \mathrm{~cm} / \mathrm{GW}\right)$ [49], CdO nanoflakes $\left(0.69 \times 10^{-3} \mathrm{~cm} / \mathrm{GW}\right)$ [50] and $\mathrm{Fe}_{2} \mathrm{O}_{3}$ hexagonal nanostructures $\left(0.82 \times 10^{-3} \mathrm{~cm} / \mathrm{GW}\right)$ [51], obtained by using the similar femtosecond laser pulses. These results indicated that BPQDs can act as good broadband optical-limiting materials in the visible range. The results are similar to the previous studies on the nonlinear absorption properties of BP nanoplatelets at $800 \mathrm{~nm}$ [52]. These nonlinear absorption properties can be well analyzed based on the band structure of BP. The bandgap of bulk BP is $\sim 0.3 \mathrm{eV}$, and the bandgap increases with the layer decreasing. With the size and layer of BPs decreasing, the quantum effects become increasingly important. Upon excitation by the photon with an energy of $1.3-2.48 \mathrm{eV}$ (equivalent to 500-900 nm), electrons cannot be promoted from the top of the valence band (VB) through direct transition but can only jump by indirect transition or through the defects level, as the photon energy is much larger than the bandgap of BP. For the energy $(2.6-4.96 \mathrm{eV})$ of two photons at $500-900 \mathrm{~nm}$, when the excitation intensity is high, electrons can be directly promoted from the top of the valence band through the two-photon absorption (TPA) process, which deduced the observed significant optical-limiting properties. 

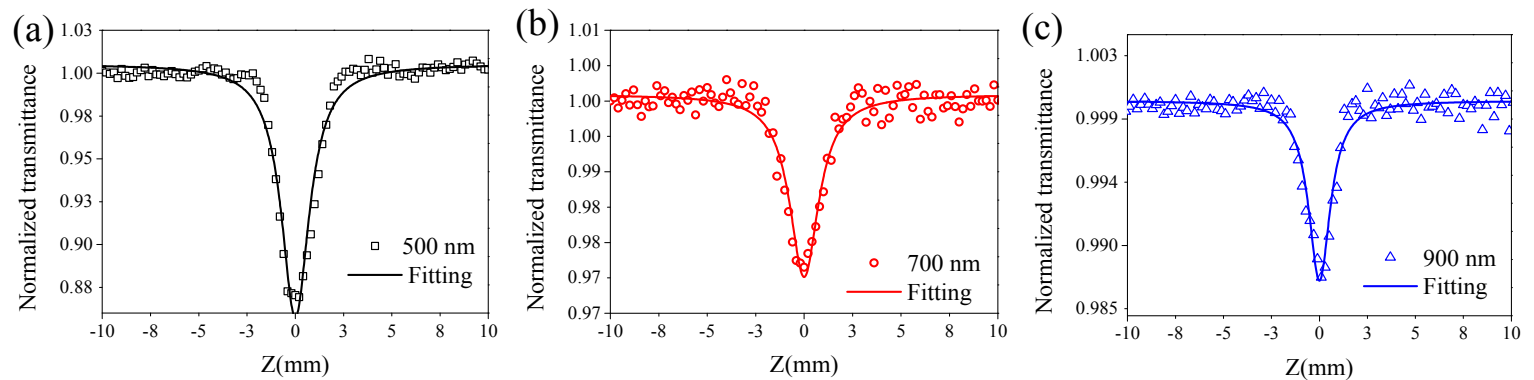

Figure 3. The open aperture Z-scan results of BPQD dispersion in NMP measured by femtosecond laser pulses with central wavelength at $500 \mathrm{~nm}(\mathbf{a}) ; 700 \mathrm{~nm}(\mathbf{b})$ and $900 \mathrm{~nm}$ (c) under the same peak power intensity of $147 \mathrm{GW} / \mathrm{cm}^{2}$, respectively.

\subsection{The Ultrafast Carrier Dynamics of BPQDs}

To better understand the possible mechanisms behind the nonlinear absorption properties in the BPQDs, transient absorption (TA) spectra as well as the single-wavelength dynamics were measured under excitation at $400 \mathrm{~nm}(3.1 \mathrm{eV})$ with a pump fluence of $16 \mu \mathrm{J} / \mathrm{cm}^{2}$. TA spectra of BPQDs in NMP at various delay times are shown in Figure $4 \mathrm{a}$. A broad negative transient differential transmission band spanning from 450 to $750 \mathrm{~nm}$ was found. The observed negative transmission change signal is commonly assigned to the excited state absorption. This result is also consistent with the previously reported photon-induced free carrier participated interband transient absorption in few-layer BP and graphene [53,54]. As mentioned above, the excitation photon energy $(3.1 \mathrm{eV})$ is much larger than the bandgap of BP. Thus, the negative transmission change signal induced by interband transient absorption could be attributed to the multiphoton absorption process. Our pump-probe results further support the nonlinear absorption behaviors observed by the Z-scan measurement in the visible range. Figure $4 \mathrm{~b}$ shows the single-wavelength dynamics probed at the wavelengths of 520 and $700 \mathrm{~nm}$. The photo-induced absorption decays probed at different wavelengths show no difference and could be well fit with a bi-exponential equation with time constants of $\tau_{1}=78 \pm 6$ ps (35\%) and $\tau_{2}=612 \pm 30 \mathrm{ps}(65 \%)$. The two characteristic time constants on the order of tens of picoseconds and hundreds of picoseconds could be attributed to the electron-phonon and slower phonon-phonon scattering during the interband transition.
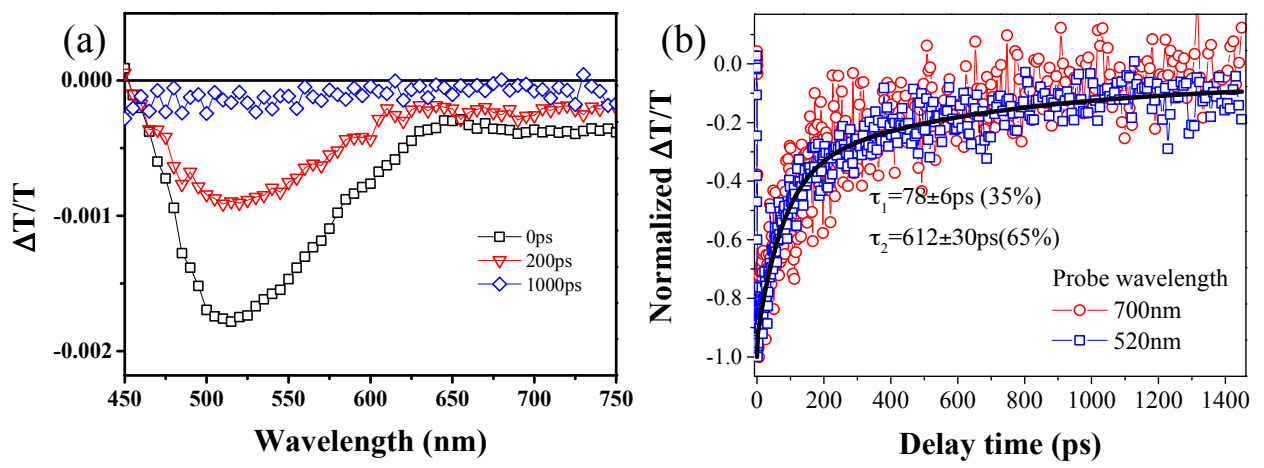

Figure 4. (a) Transient absorption spectra of BPQDs in NMP at the different delay times under excitation at $400 \mathrm{~nm}$; (b) Single-wavelength dynamics at probed at 525 and $700 \mathrm{~nm}$, measured by femtosecond pump-probe technique.

\subsection{The Nonlinar Optical Properties of BPQDs in Near-IR Range}

We also investigated the nonlinear absorption responses of BPQDs in NMP at 1050, $1350 \mathrm{~nm}$, respectively. The open aperture Z-scan measurement results under different excitation peak power 
intensities at the focal point are shown in Figure 5. The normalized transmittance gradually increased with the approaching of the BPQD sample with respect to the focal point $(Z=0)$, indicating that the absorption of BPQDs becomes saturated with the increase of the incident pump intensity. This is well known as the saturable absorption behavior. As shown in the absorption spectra, there is a strong absorption band in the near-IR range. The observed saturable absorption activity can be ascribed to the ground-state bleaching induced by photo-excitation. Figure 5 shows the power-dependent saturable absorption. It can be seen that the peaks of the open Z-scan curves increased with the increasing input power intensity. These results further confirm that the saturable absorption responses indeed originate from the intrinsic optical absorption effects in BPQDs other than from artifacts such as sample damage or contamination. When the excitation intensity increased further until it reached the photo-damage threshold, the reverse saturable absorption effect was not observed. These results can be ascribed to smaller two-photon absorption coefficients of BPQDs at the near-IR spectra range, which are not enough to overcome the ground-state bleaching. These results are consistent with the wavelength dependence of the two-photon absorption coefficient in the visible spectra range: the two-photon absorption coefficient decreased with the increasing wavelength.
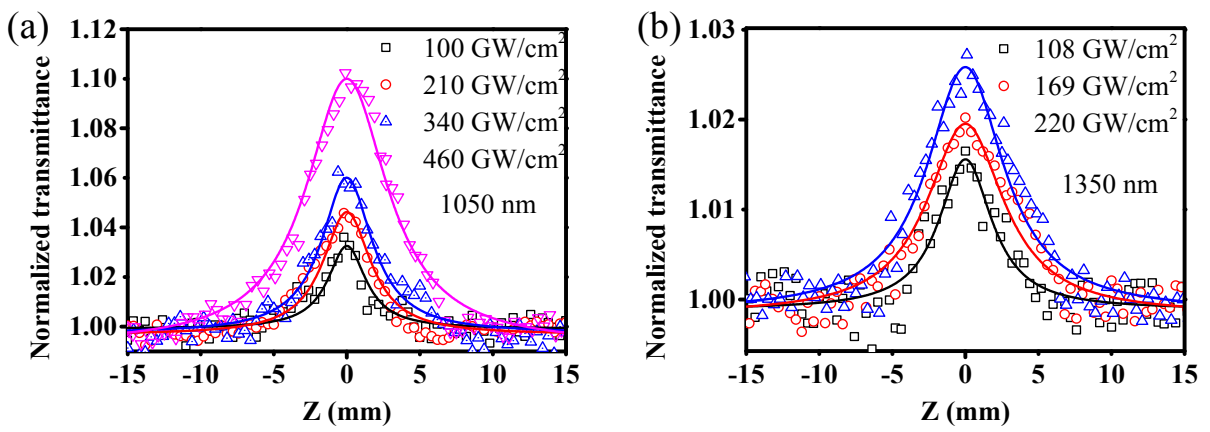

Figure 5. The open aperture Z-scan results of BPQDs at $1050 \mathrm{~nm}$ (a) and $1350 \mathrm{~nm}$ (b) under different excitation peak power intensities at the focal point.

Similar measurements have also been performed at $1550 \mathrm{~nm}$. Figure 6 shows the Z-scan curve of BPQDs under the excitation peak power intensity of $38 \mathrm{GW} / \mathrm{cm}^{2}$ and the corresponding fitting curve of saturable absorption. Based on the relation between the laser beam spot size and the relative separation, the nonlinear saturable absorption curve could be derived (Figure 6b). The onset of saturation intensity was around $2.5 \mathrm{GW} / \mathrm{cm}^{2}$ and the modulation depth was $8.2 \%$ at $1550 \mathrm{~nm}$. Therefore, the measured results indicate that the as-prepared BPQDs can be used as a saturable absorber for ultrafast laser pulse generation in near-IR fiber lasers.
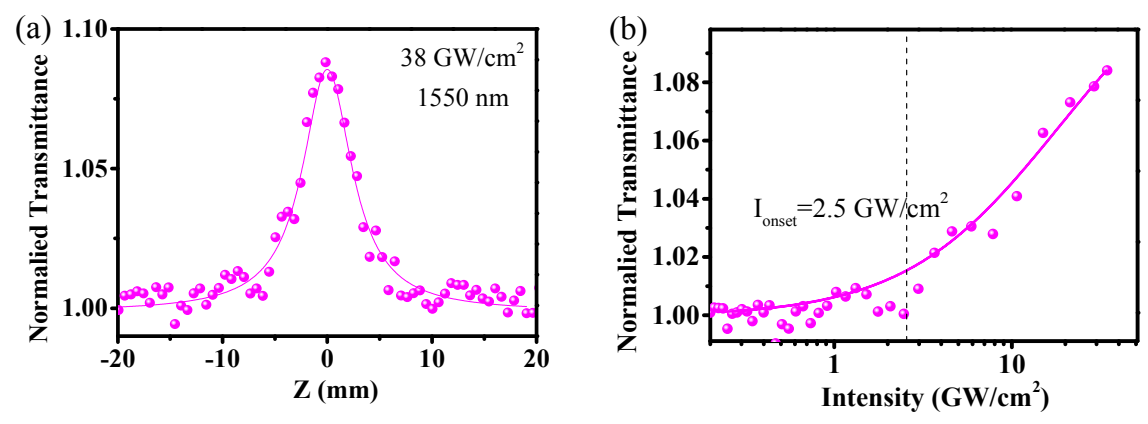

Figure 6. The open aperture Z-scan results of BPQDs at $1550 \mathrm{~nm}$ (a) and its corresponding saturable absorption curve (b). 


\section{Materials and Methods}

\subsection{Synthesis of $B P Q D$ s}

NMP was used to isolate the BPQDs from the bulk BP crystal. First, the BP bulk crystal $(99.998 \%$, purchased from smart elements) was pulverized to prepare the BP powder. The dispersed suspension of BPQDs was then prepared by ultrasound probe sonication followed by ice bath sonication of bulk BP powder in 1-methyl-2-pyrro-lidone (NMP, 99.5\% anhydrous, purchased from Aladdin Reagents). Then $25 \mathrm{mg}$ of the BP powder was dispersed into $25 \mathrm{~mL}$ of NMP in a $50 \mathrm{~mL}$ sealed conical tube and sonicated with a sonic tip for $3 \mathrm{~h}$ at the power of $1200 \mathrm{~W}$. The ultrasonic frequency ranged from 19 to $25 \mathrm{kHz}$ and the ultrasound probe worked $2 \mathrm{~s}$ with an interval of $4 \mathrm{~s}$. Afterwards, an ultrasonic bath was adopted to sonicate the dispersion consecutively for another $10 \mathrm{~h}$ at the power of $300 \mathrm{~W}$. During the sonication, the temperature of the sample solution was kept and monitored below $277 \mathrm{~K}$ in an ice bath. The dispersion was then centrifuged at the speed of $7000 \mathrm{rpm}$ for $20 \mathrm{~min}$. The supernatant containing BPQDs was collected. To further increase the concentration of the BPQDs dispersion, the collected solution was centrifuged for $20 \mathrm{~min}$ at the speed of 12,000 rpm. The resulting supernatant was collected and was kept in a sealed tube for further tests.

Transmission electron microscopy (TEM) measurements were conducted to examine the morphology of the as-prepared BPQDs. The TEM images were taken on the Tecnai G2 F20 S-Twin transmission electron microscope at an acceleration voltage of $200 \mathrm{kV}$. The absorption spectra were taken by a Shimadzu UV3600 spectroscopy with QS-grade quartz cuvette at room temperature.

\subsection{Nonlinear Optical Property Measured by Z-Scan Technique}

The open aperture Z-scan measurements were performed by using an optical parametric amplifier (TOPAS-Prime) pumped by a mode-locked Ti:sapphire oscillator seeded regenerative amplifier (Spectra-Physics Spitfire Ace), which gives output laser pulses with tunable central wavelength from UV to near-IR range, pulse duration of $\sim 120$ fs and repetition rate of $1 \mathrm{kHz}$. The laser beam was focused onto the sample with a beam radius of $\sim 23 \mu \mathrm{m}$. Samples were prepared in the quartz cuvette with the optical path length of $1 \mathrm{~mm}$. The transmittance of the samples was measured as a function of input intensity, which was varied by moving the samples in and out of beam focus along the z-axis. At least five cycles were repeated to reduce the experimental error and the average was calculated to plot the Z-scan curves.

\subsection{Pump-Probe and Transient Absorption Spectra Mansurement}

In our experiments, a Ti:sapphire oscillator seeded regenerative amplifier laser system (Spectra Physics Spitfire Ace) with output pulse energy of $2 \mathrm{~mJ}$ at $800 \mathrm{~nm}$ and a repetition rate of $1 \mathrm{kHz}$ was used as the source of the pump and beam pulses. The detailed experimental setup is described in Figure 7. The $800 \mathrm{~nm}$ laser beam was split into two portions. One portion passes through a BBO crystal to generate the $400 \mathrm{~nm}$ pump beam by second harmonic generation. The other portion of the $800 \mathrm{~nm}$ beam was used to generate white light continuum in a $2.5 \mathrm{~mm}$ sapphire plate. The white light beam was split into two portions: one as the probe and another as the reference. The pump beam was focused onto the sample with a beam size with $300 \mu \mathrm{m}$ in diameter and overlapped with the smaller probe beam (100 $\mu \mathrm{m}$ in diameter). The probe beam is collected after passing through the sample and its intensity is monitored by a photodiode as the detector. Both the detectors of probe and reference beam are connected to the lock-in amplifier to correct the pulse-to-pulse intensity fluctuations. The time delay between the pump and probe pulse was varied by a computer-controlled translation stage. The pump beam was modulated by an optical chopper at the frequency of $500 \mathrm{~Hz}$. In a single-wavelength dynamics scan, a fixed probe wavelength is picked and the transmittance change of probe with pump and without pump $(\Delta \mathrm{T} / \mathrm{T})$ was monitored as a function of the various delay between pump and probe beam. The transient absorption spectra at different delay time were measured by passing the probe through a monochromator before detector. 


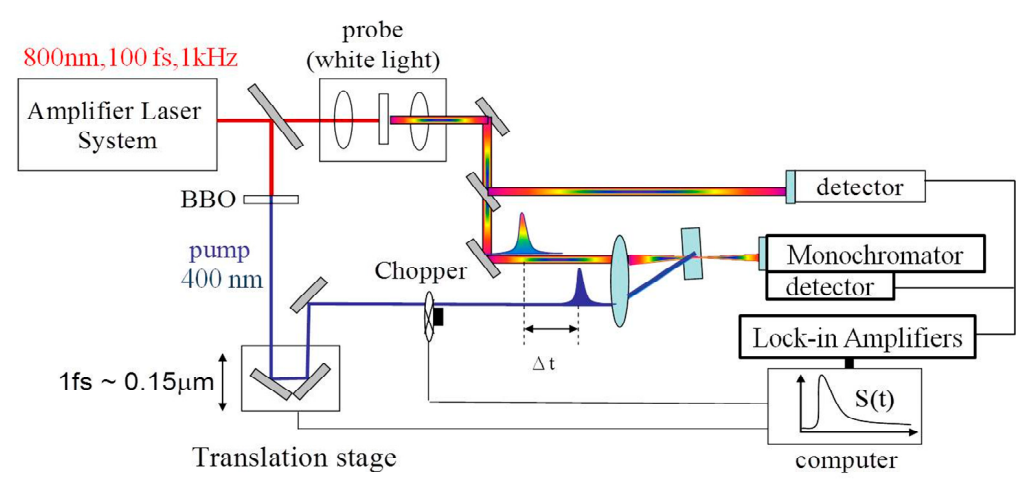

Figure 7. Experimental setup of pump-probe and transient absorption measurements.

\section{Conclusions}

In conclusion, BPQDs were fabricated by a simple solvent exfoliation method. The nonlinear absorption properties were investigated with Z-scan measurements using femtosecond laser pulses at 500, 700, 900, 1050, 1350 and $1550 \mathrm{~nm}$. These ultrasmall BPQS in NMP suspension exhibited broadband optical-limiting activities in the visible range. The nonlinear absorption coefficient was found to be $(7.49 \pm 0.23) \times 10^{-3},(1.68 \pm 0.078) \times 10^{-3}$ and $(0.81 \pm 0.03) \times 10^{-3} \mathrm{~cm} / \mathrm{GW}$ for 500,700 and $900 \mathrm{~nm}$, respectively. Femtosecond pump-probe measurements performed on the BPQD suspension further support that nonlinear absorption due to two-photon absorption played an important role in the observed optical-limiting activities of the BPQDs. Furthermore, saturable absorption due to ground-state bleaching was found in BPQDs by photo-excitation at 1050, 1350 and $1550 \mathrm{~nm}$. Our results will provide the basis for applications of BPQDs in broadband optoelectronic devices such as optical limiters in the visible range or saturable absorbers in the near-IR range.

Acknowledgments: We thank the financial support from the Guangdong Innovative Research Team Program of China (Grant No. 201101C0105067115), the Young Scientists Fund of the National Natural Science Foundation of China (Grant No. 51603069), the National Natural Science Foundation of China (Grant No. 21673155), the Scientific Research Staring Natural Science Foundation of Guangdong Province, China (Grant No. 2016A030310432), the Fundamental Research Funds for the Central Universities and the China Postdoctoral Science Foundation.

Author Contributions: For research articles with several authors, a short paragraph specifying their individual contributions must be provided. The following statements should be used “X.F. Jiang, Z. Zeng, S. Li, F. Huang, and Q.-H. Xu conceived and designed the experiments; X.F. Jiang and Z. Zeng performed the experiments, analyzed the data as well as wrote the paper; Z. Guo and H. Zhang contributed the materials; X.F. Jiang, Z. Zeng and Q.-H. Xu wrote the paper. All the authors have contributed substantially to the work reported.

Conflicts of Interest: The authors declare no conflict of interest.

\section{References}

1. Novoselov, K.S.; Geim, A.K.; Morozov, S.V.; Jiang, D.; Zhang, Y.; Dubonos, S.V.; Grigorieva, I.V.; Firsov, A.A. Electric field effect in atomically thin carbon films. Science 2004, 306, 666-669. [CrossRef] [PubMed]

2. Dang, X.N.; Yi, H.J.; Ham, M.H.; Qi, J.F.; Yun, D.S.; Ladewski, R.; Strano, M.S.; Hammond, P.T.; Belcher, A.M. Virus-templated self-assembled single-walled carbon nanotubes for highly efficient electron collection in photovoltaic devices. Nat. Nanotechnol. 2011, 6, 377-384. [CrossRef] [PubMed]

3. Miao, X.C.; Tongay, S.; Petterson, M.K.; Berke, K.; Rinzler, A.G.; Appleton, B.R.; Hebard, A.F. High efficiency graphene solar cells by chemical doping. Nano Lett. 2012, 12, 2745-2750. [CrossRef] [PubMed]

4. Kim, K.S.; Zhao, Y.; Jang, H.; Lee, S.Y.; Kim, J.M.; Kim, K.S.; Ahn, J.H.; Kim, P.; Choi, J.Y.; Hong, B.H. Large-scale pattern growth of graphene films for stretchable transparent electrodes. Nature 2009, 457, 706-710. [CrossRef] [PubMed]

5. Sanders, S.; Cabrero-Vilatela, A.; Kidambi, P.R.; Alexander-Webber, J.A.; Weijtens, C.; Braeuninger-Weimer, P.; Aria, A.I.; Qasim, M.M.; Wilkinson, T.D.; Robertson, J.; et al. Engineering high charge transfer n-doping of graphene electrodes and its application to organic electronics. Nanoscale 2015, 7, 13135-13142. [CrossRef] [PubMed] 
6. Castro Neto, A.H.; Novoselov, K. Two-dimensional crystals: Beyond graphene. Mater. Express 2011, 1, $10-17$. [CrossRef]

7. Lim, G.K.; Chen, Z.L.; Clark, J.; Goh, R.G.S.; Ng, W.H.; Tan, H.W.; Friend, R.H.; Ho, P.K.H.; Chua, L.L. Giant broadband nonlinear optical absorption response in dispersed graphene single sheets. Nat. Photonics 2011, 5, 554-560. [CrossRef]

8. Feng, M.; Zhan, H.B.; Chen, Y. Nonlinear optical and optical limiting properties of graphene families. Appl. Phys. Lett. 2010, 96, 033107. [CrossRef]

9. Jiang, X.F.; Polavarapu, L.; Neo, S.T.; Venkatesan, T.; Xu, Q.H. Graphene oxides as tunable broadband nonlinear optical materials for femtosecond laser pulses. J. Phys. Chem. Lett. 2012, 3, 785-790. [CrossRef] [PubMed]

10. Jiang, X.-F.; Polavarapu, L.; Zhu, H.; Ma, R.; Xu, Q.-H. Flexible, robust and highly efficient broadband nonlinear optical materials based on graphene oxide impregnated polymer sheets. Photonic Res. 2015, 3, A87-A91. [CrossRef]

11. Wang, K.P.; Feng, Y.Y.; Chang, C.X.; Zhan, J.X.; Wang, C.W.; Zhao, Q.Z.; Coleman, J.N.; Zhang, L.; Blau, W.J.; Wang, J. Broadband ultrafast nonlinear absorption and nonlinear refraction of layered molybdenum dichalcogenide semiconductors. Nanoscale 2014, 6, 10530-10535. [CrossRef] [PubMed]

12. Wang, S.X.; Yu, H.H.; Zhang, H.J.; Wang, A.Z.; Zhao, M.W.; Chen, Y.X.; Mei, L.M.; Wang, J.Y. Broadband few-layer $\mathrm{MoS}_{2}$ saturable absorbers. Adv. Mater. 2014, 26, 3538-3544. [CrossRef] [PubMed]

13. Khazaeizhad, R.; Kassani, S.H.; Jeong, H.; Yeom, D.I.; Oh, K. Mode-locking of er-doped fiber laser using a multilayer MoS2 thin film as a saturable absorber in both anomalous and normal dispersion regimes. Opt. Express 2014, 22, 23732-23742. [CrossRef] [PubMed]

14. Xia, H.D.; Li, H.P.; Lan, C.Y.; Li, C.; Zhang, X.X.; Zhang, S.J.; Liu, Y. Ultrafast erbium-doped fiber laser mode-locked by a cvd-grown molybdenum disulfide $\left(\mathrm{MoS}_{2}\right)$ saturable absorber. Opt. Express 2014, 22, 17341-17348. [CrossRef] [PubMed]

15. Zhang, X.; Xie, H.M.; Liu, Z.D.; Tan, C.L.; Luo, Z.M.; Li, H.; Lin, J.D.; Sun, L.Q.; Chen, W.; Xu, Z.C.; et al. Black phosphorus quantum dots. Angew. Chem. Int. Ed. 2015, 54, 3653-3657. [CrossRef] [PubMed]

16. Churchill, H.H.; Jarillo-Herrero, P. Two-dimensional crystals phosphorus joins the familly. Nat. Nanotechnol. 2014, 9, 330-331. [CrossRef] [PubMed]

17. Li, L.K.; Yu, Y.J.; Ye, G.J.; Ge, Q.Q.; Ou, X.D.; Wu, H.; Feng, D.L.; Chen, X.H.; Zhang, Y.B. Black phosphorus field-effect transistors. Nat. Nanotechnol. 2014, 9, 372-377. [CrossRef] [PubMed]

18. Xia, F.N.; Wang, H.; Jia, Y.C. Rediscovering black phosphorus as an anisotropic layered material for optoelectronics and electronics. Nat. Commun. 2014, 5, 4458. [CrossRef] [PubMed]

19. Reich, E.S. Phosphorene excites materials scientists. Nature 2014, 506, 19. [CrossRef] [PubMed]

20. Castellanos-Gomez, A.; Vicarelli, L.; Prada, E.; Island, J.O.; Narasimha-Acharya, K.L.; Blanter, S.I.; Groenendijk, D.J.; Buscema, M.; Steele, G.A.; Alvarez, J.V.; et al. Isolation and characterization of few-layer black phosphorus. 2D Mater. 2014, 1, 025001. [CrossRef]

21. Kang, J.; Wood, J.D.; Wells, S.A.; Lee, J.H.; Liu, X.L.; Chen, K.S.; Hersam, M.C. Solvent exfoliation of electronic-grade, two-dimensional black phosphorus. ACS Nano 2015, 9, 3596-3604. [CrossRef] [PubMed]

22. Brent, J.R.; Savjani, N.; Lewis, E.A.; Haigh, S.J.; Lewis, D.J.; O’Brien, P. Production of few-layer phosphorene by liquid exfoliation of black phosphorus. Chem. Commun. 2014, 50, 13338-13341. [CrossRef] [PubMed]

23. Liu, X.L.; Wood, J.D.; Chen, K.S.; Cho, E.; Hersam, M.C. In Situ thermal decomposition of exfoliated two-dimensional black phosphorus. J. Phys. Chem. Lett. 2015, 6, 773-778. [CrossRef] [PubMed]

24. Koenig, S.P.; Doganov, R.A.; Schmidt, H.; Neto, A.H.C.; Ozyilmaz, B. Electric field effect in ultrathin black phosphorus. Appl. Phys. Lett. 2014, 104, 103106. [CrossRef]

25. Dai, J.; Zeng, X.C. Bilayer phosphorene: Effect of stacking order on bandgap and its potential applications in thin-film solar cells. J. Phys. Chem. Lett. 2014, 5, 1289-1293. [CrossRef] [PubMed]

26. Deng, Y.X.; Luo, Z.; Conrad, N.J.; Liu, H.; Gong, Y.J.; Najmaei, S.; Ajayan, P.M.; Lou, J.; Xu, X.F.; Ye, P.D. Black phosphorus-monolayer MoS2 van der waals heterojunction p-n diode. ACS Nano 2014, 8, 8292-8299. [CrossRef] [PubMed]

27. Zhu, W.N.; Yogeesh, M.N.; Yang, S.X.; Aldave, S.H.; Kim, J.S.; Sonde, S.; Tao, L.; Lu, N.S.; Akinwande, D. Flexible black phosphorus ambipolar transistors, circuits and am demodulator. Nano Lett. 2015, 15, 1883-1890. [CrossRef] [PubMed] 
28. Zhao, S.J.; Kang, W.; Xue, J.M. The potential application of phosphorene as an anode material in li-ion batteries. J. Mater. Chem. A 2014, 2, 19046-19052. [CrossRef]

29. Wang, Z.H.; Jia, H.; Zheng, X.Q.; Yang, R.; Wang, Z.F.; Ye, G.J.; Chen, X.H.; Shan, J.; Feng, P.X.L. Black phosphorus nanoelectromechanical resonators vibrating at very high frequencies. Nanoscale 2015, 7, 877-884. [CrossRef] [PubMed]

30. Song, Y.; Chen, S.; Zhang, Q.; Li, L.; Zhao, L.; Zhang, H.; Tang, D. Vector soliton fiber laser passively mode locked by few layer black phosphorus-based optical saturable absorber. Opt. Express 2016, 24, 25933-25942. [CrossRef] [PubMed]

31. Sotor, J.; Sobon, G.; Kowalczyk, M.; Macherzynski, W.; Paletko, P.; Abramski, K.M. Ultrafast thulium-doped fiber laser mode locked with black phosphorus. Opt. Lett. 2015, 40, 3885-3888. [CrossRef] [PubMed]

32. Luo, Z.-C.; Liu, M.; Guo, Z.-N.; Jiang, X.-F.; Luo, A.-P.; Zhao, C.-J.; Yu, X.-F.; Xu, W.-C.; Zhang, H. Microfiber-based few-layer black phosphorus saturable absorber for ultra-fast fiber laser. Opt. Express 2015, 23, 20030-20039. [CrossRef] [PubMed]

33. Li, D.; Jussila, H.; Karvonen, L.; Ye, G.; Lipsanen, H.; Chen, X.; Sun, Z. Polarization and thickness dependent absorption properties of black phosphorus: New saturable absorber for ultrafast pulse generation. Sci. Rep. 2015, 5, 15899. [CrossRef] [PubMed]

34. Sotor, J.; Sobon, G.; Macherzynski, W.; Paletko, P.; Abramski, K.M. Black phosphorus saturable absorber for ultrashort pulse generation. Appl. Phys. Lett. 2015, 107, 051108. [CrossRef]

35. Su, X.; Wang, Y.; Zhang, B.; Zhao, R.; Yang, K.; He, J.; Hu, Q.; Jia, Z.; Tao, X. Femtosecond solid-state laser based on a few-layered black phosphorus saturable absorber. Opt. Lett. 2016, 41, 1945-1948. [CrossRef] [PubMed]

36. Liu, H.; Neal, A.T.; Zhu, Z.; Luo, Z.; Xu, X.F.; Tomanek, D.; Ye, P.D.D. Phosphorene: An unexplored 2D semiconductor with a high hole mobility. ACS Nano 2014, 8, 4033-4041. [CrossRef] [PubMed]

37. Tran, V.; Soklaski, R.; Liang, Y.F.; Yang, L. Layer-controlled band gap and anisotropic excitons in few-layer black phosphorus. Phys. Rev. B 2014, 89, 235319. [CrossRef]

38. Rodin, A.S.; Carvalho, A.; Castro Neto, A.H. Strain-induced gap modification in black phosphorus. Phys. Rev. Lett. 2014, 112, 176801. [CrossRef] [PubMed]

39. Jiang, J.W.; Park, H.S. Analytic study of strain engineering of the electronic bandgap in single-layer black phosphorus. Phys. Rev. B 2015, 91, 235118. [CrossRef]

40. Wang, T.; Liu, Y.; Guo, Q.; Zhang, B.; Sheng, K.; Li, C.; Yin, Y. Tunable bandgap of monolayer black phosphorus by using vertical electric field: A dft study. J. Korean Phys. Soc. 2015, 66, 1031-1034. [CrossRef]

41. Mamidala, V.; Polavarapu, L.; Balapanuru, J.; Loh, K.P.; Xu, Q.H.; Ji, W. Enhanced nonlinear optical responses in donor-acceptor ionic complexes via photo induced energy transfer. Opt. Express 2010, 18, 25928-25935. [CrossRef] [PubMed]

42. Bonaccorso, F.; Sun, Z.; Hasan, T.; Ferrari, A.C. Graphene photonics and optoelectronics. Nat. Photonics 2010, 4, 611-622. [CrossRef]

43. Bonaccorso, F.; Sun, Z.P. Solution processing of graphene, topological insulators and other 2D crystals for ultrafast photonics. Opt. Mater. Express 2014, 4, 63-78. [CrossRef]

44. Sun, Z.; Xie, H.; Tang, S.; Yu, X.-F.; Guo, Z.; Shao, J.; Zhang, H.; Huang, H.; Wang, H.; Chu, P.K. Ultrasmall black phosphorus quantum dots:Synthesis and use as photothermal agents. Angew. Chem. Int. Ed. 2105, 54, 11526-11530. [CrossRef] [PubMed]

45. Joshua, O.I.; Gary, A.S.; Herre, S.J.v.d.Z.; Andres, C.-G. Environmental instability of few-layer black phosphorus. 2D Mater. 2015, 2, 011002.

46. Wood, J.D.; Wells, S.A.; Jariwala, D.; Chen, K.-S.; Cho, E.; Sangwan, V.K.; Liu, X.; Lauhon, L.J.; Marks, T.J.; Hersam, M.C. Effective passivation of exfoliated black phosphorus transistors against ambient degradation. Nano Lett. 2014, 14, 6964-6970. [CrossRef] [PubMed]

47. Low, T.; Rodin, A.; Carvalho, A.; Jiang, Y.; Wang, H.; Xia, F.; Neto, A.C. Tunable optical properties of multilayer black phosphorus thin films. Phys. Rev. B 2014, 90, 075434. [CrossRef]

48. Lu, S.B.; Miao, L.L.; Guo, Z.N.; Qi, X.; Zhao, C.J.; Zhang, H.; Wen, S.C.; Tang, D.Y.; Fan, D.Y. Broadband nonlinear optical response in multi-layer black phosphorus: An emerging infrared and mid-infrared optical material. Opt. Express 2015, 23, 11183-11194. [CrossRef] [PubMed] 
49. He, G.S.; Yong, K.-T.; Zheng, Q.; Sahoo, Y.; Baev, A.; Ryasnyanskiy, A.I.; Prasad, P.N. Multi-photon excitation properties of cdse quantum dots solutions and optical limiting behavior in infrared range. Opt. Express 2007, 15, 12818-12833. [CrossRef] [PubMed]

50. Thomas, P.; Sreekanth, P.; Philip, R.; Abraham, K.E. Morphology dependent nanosecond and ultrafast optical power limiting of cdo nanomorphotypes. RSC Adv. 2015, 5, 35017-35025. [CrossRef]

51. Thomas, P.; Sreekanth, P.; Abraham, K.E. Nanosecond and ultrafast optical power limiting in luminescent $\mathrm{Fe}_{2} \mathrm{O}_{3}$ hexagonal nanomorphotype. J. Appl. Phys. 2015, 117, 053103. [CrossRef]

52. Zheng, X.; Chen, R.; Shi, G.; Zhang, J.; Xu, Z.; Jiang, T. Characterization of nonlinear properties of black phosphorus nanoplatelets with femtosecond pulsed z-scan measurements. Opt. Lett. 2015, 40, 3480-3483. [CrossRef] [PubMed]

53. Suess, R.J.; Jadidi, M.M.; Murphy, T.E.; Mittendorff, M. Carrier dynamics and transient photobleaching in thin layers of black phosphorus. Appl. Phys. Lett. 2015, 107, 081103. [CrossRef]

54. Kadi, F.; Winzer, T.; Malic, E.; Knorr, A.; Göttfert, F.; Mittendorff, M.; Winnerl, S.; Helm, M. Microscopic description of intraband absorption in graphene: The occurrence of transient negative differential transmission. Phys. Rev. Lett. 2014, 113, 035502. [CrossRef] [PubMed]

(C) 2017 by the authors. Licensee MDPI, Basel, Switzerland. This article is an open access article distributed under the terms and conditions of the Creative Commons Attribution (CC BY) license (http:/ / creativecommons.org/licenses/by/4.0/). 